\title{
Qualitative Study on Male Nursing Students' Cognition of Nine-Valent Preventive Vaccine
}

\author{
Tiantian Jia', Junjuan Zhang2*, Jiajia Li², Lilu Tian'1, Yufeng Liư1, Wenjing Yu², Ruijuan Han' \\ ${ }^{1}$ School of Nursing and Health, Henan University, Kaifeng 475004, Henan Province, China \\ ${ }^{2}$ Henan Provincial People's Hospital, Zhengzhou 450000, Henan Province, China \\ *Corresponding author: Junjuan Zhang, 383583496@qq.com
}

\begin{abstract}
Objective: To investigate the cognition degree of male nursing students to HPV and nine-valent vaccine and the influencing factors of male willingness to receive nine-valent HPV vaccine. Methods: Qualitative research semi-structured interview was conducted on male nursing students, and phenomenological research method was used to record, ran scribe, encode and classify the interview data and extract the topic. The number of interviews was determined according to the information saturation. Results: Seven themes were summarized in this study, which were mainly divided into two aspects. The promotion of vaccination includes two aspects: self-benefit and benefit of others. The prevention of vaccination mainly includes low awareness rate, feminization of vaccine information, high cost and safety and effectiveness. Conclusion: Male nursing students have low cognition degree and inoculation intention to human papillomavirus and nine-valent vaccine, so it is necessary to further strengthen publicity and carry out college education to improve vaccination.
\end{abstract}

Keywords: Male nursing students; HPV; Nine-valent vaccine; Intention to vaccinate; Qualitative research

Publication date: September 2021; Online publication: September 30, 2021

\section{Introduction}

Human papillomavirus (HPV) is a common reproductive tract infection virus. High risk HPV (HR-HPV) persistent infection can cause cervical cancer, and is also associated with the occurrence of vaginal cancer, vulvar cancer, anal cancer, penile cancer and oropharyngeal cancer. It is an important public health problem in the world, especially in developing countrie ${ }^{[1]}$. HPV infection is the most common sexually transmitted disease, usually infecting sexually active young people ${ }^{[2]}$. In 2014 , WHO has shown that HPV vaccination can reduce the risk of sexually transmitted diseases, and thus reduce the incidence rate of cervical cancer in women ${ }^{[3]}$. At present, the research on HPV vaccine mostly uses quantitative research to describe the current situation and influencing factors, pays more attention to women, and lacks attention to men, especially focusing on the qualitative research on male nursing students' cognition and vaccination intention. Male nursing students are future health professionals, most of whom are aged from 16 to 26. They meet the target population of nine valent HPV vaccination. They play an important role in providing the public with HPV screening and protection methods ${ }^{[4,5]}$. The purpose of this study is to understand the influencing factors of male nursing students' awareness of HPV virus and willingness to vaccinate with nine valent HPV vaccine, so as to provide reference basis for smoothly carrying out effective cervical cancer intervention measures in the future and promoting the popularization of nine valent HPV vaccine in China.

\section{Research object and method}

\subsection{Research object}

To interview 12 male nursing students from 3 colleges and universities in Henan Province from June to 
August 2020. Inclusion criteria:

(1) Full time male nursing students enrolled in the national uniform.

(2) Informed and agreed.

(3) Good language communication skills and clear language expression.

Exclusion criteria: Researchers who have participated in similar research in recent half a year. The determination of sample size is based on the repeated occurrence of respondents' data and no new topics in data analysis, that is, the data will stop after reaching saturation ${ }^{[6]}$.

A total of 12 male nursing students were included in this study, which were numbered with English letters A L. Among them, there are 1 technical secondary school student, 2 junior college students, 4 undergraduates and 5 postgraduates. See Table 1. for general information of respondents. The study meets the requirements of the Helsinki declaration.

Table 1. General information of 12 respondents

\begin{tabular}{|c|c|c|c|c|c|c|c|}
\hline Letter & $\begin{array}{c}\text { Educational } \\
\text { level }\end{array}$ & $\begin{array}{c}\text { Age } \\
\text { (years) }\end{array}$ & $\begin{array}{c}\text { Are there } \\
\text { any brothers } \\
\text { / sisters }\end{array}$ & $\begin{array}{c}\text { Have a } \\
\text { history of } \\
\text { asexual life }\end{array}$ & $\begin{array}{l}\text { Monthly } \\
\text { living } \\
\text { expenses }\end{array}$ & $\begin{array}{c}\text { HPV } \\
\text { vaccination } \\
\text { history }\end{array}$ & $\begin{array}{c}\text { HPV } \\
\text { cognitive } \\
\text { sources }\end{array}$ \\
\hline A & $\begin{array}{l}\text { Secondary } \\
\text { specialized } \\
\text { school }\end{array}$ & 18 & No & Yes & 2000 & Nothing & Internet \\
\hline B & Junior college & 20 & No & Nothing & 1300 & Nothing & Classmate \\
\hline $\mathrm{C}$ & Junior college & 22 & Yes & Nothing & 2600 & Nothing & $\begin{array}{c}\text { Newspapers } \\
\text { and } \\
\text { periodicals }\end{array}$ \\
\hline $\mathrm{D}$ & Undergraduate & 21 & No & Yes & 1500 & Nothing & Friend \\
\hline $\mathrm{E}$ & Undergraduate & 19 & Yes & Nothing & 1800 & Nothing & Classmate \\
\hline $\mathrm{F}$ & Undergraduate & 22 & Yes & Nothing & 1400 & Nothing & $\begin{array}{c}\text { Brochures, } \\
\text { students }\end{array}$ \\
\hline $\mathrm{G}$ & Undergraduate & 24 & Yes & Yes & 3000 & Nothing & Friend \\
\hline $\mathrm{H}$ & Master & 24 & No & Yes & 2000 & Nothing & $\begin{array}{l}\text { Students, } \\
\text { websites }\end{array}$ \\
\hline I & Master & 26 & No & Yes & 2400 & Nothing & $\begin{array}{l}\text { Classmates } \\
\text { and doctors }\end{array}$ \\
\hline $\mathrm{J}$ & Master & 24 & Yes & Yes & 2000 & Nothing & Internet \\
\hline $\mathrm{K}$ & Master & 25 & No & Yes & 3600 & Nothing & Lecture \\
\hline $\mathrm{L}$ & Master & 23 & Yes & Yes & 2300 & Nothing & Classmate \\
\hline
\end{tabular}




\subsection{Research methods}

Guided by the phenomenological method of qualitative research, this study collected data in the form of face-to-face interview and recorded simultaneously. Literature review was conducted before the interview. By consulting 2 gynecologists, 1 doctor of nursing and 2 gynecological clinical nursing experts, the interview outline was formulated as the interview guide and continuously improved during the interview. Male nursing students who met the inclusion criteria were introduced to the purpose, significance and time of the study, and their consent was obtained on the recording. In order to protect privacy, the research results are anonymous. Three male nursing students were selected by objective sampling method for pre interview to determine the final interview outline.

The interview outline includes:

(1) What do you think are the effects of nine valent HPV vaccine?

(2) What do you think of the risk of HPV infection?

(3) Do you think men should be vaccinated with nine valent HPV vaccine? Why?

(4) What sources have you heard of HPV and nine valent HPV vaccine?

(5) What do you want to know about the nine valent HPV vaccine?

Record the interview truthfully, listen carefully and observe the emotional changes of the respondents. The interview time of each interviewee is $30 \sim 50 \mathrm{~min}$. the interview recordings and relevant written materials are classified and kept to maintain their integrity. Sort out the data and input the recorded data into the computer for transcription within 24 hours.

\subsection{Data analysis}

The interview data are analyzed and sorted out by Colaizzi 7 step analysis method, and the interview records are carefully read to extract meaningful statements ${ }^{[7]}$. Encode, collect and extract recurring ideas. Return the ambiguous analysis results to the respondents for verification, listen to the opinions of the respondents and form the final theme.

\section{Results}

\subsection{Factors that promote participants to receive nine valent HPV vaccine \\ 3.1.1. Self-benefit}

Vaccination with nine valent HPV vaccine is an effective intervention for male nursing students themselves. Some studies have pointed out that HPV infection is one of the most important factors of penile cancer ${ }^{[1]}$. Among genital cancers with positive human papillomavirus (HPV) DNA, HPV-16 is the most common type in men, followed by HPV-18. The nine valent HPV vaccine can reduce the incidence rate of HPV above $6,11,16$ and 18 of the disease, and has a preventive effect on diseases with 96.7\% HPV 31, 33, 45, 52 and 45. It can reduce the incidence of malignant tumor in cervical, vulva, vagina, anus and penis, which are covered by HPV subtype ${ }^{[8]}$. Participant H: "I know nine-valent vaccine injection mainly aimed at women, also heard that many men will HPV infection, HPV in addition to lead to cervical cancer, and the incidence of anal cancer and prostate cancer as well as related, from the vaccine research and development to the public after a series of clinical trials, don't worry about it too much side effect, cost nothing for your health. It's a very small investment." Participant D: "Now you spend thousands of yuan on vaccination, and maybe you will save hundreds of thousands of yuan for chemotherapy in the future. It's really costeffective." 


\subsubsection{Benefits to others}

Vaccination with nine valent HPV vaccine not only benefits future spouses and families, but also actively promotes the vaccination behavior of peers. HPV infection is mainly caused by sexual transmission. It is a sexually transmitted disease with strong infectivity, high infection rate and high recurrence rate. Participant $\mathrm{K}$ : "I was concerned about the nine valent HPV vaccine, because my girlfriend, the incidence rate of cervical cancer is too high now, showing a younger trend. I have been urging my girlfriend to inoculate. If we two can reduce the incidence rate by vaccination, that is very necessary." Prevention and intervention of HPV must be carried out between men and women, so that the incidence rate of cervical cancer can be reduced. HPV vaccine can protect its own sexual partners while protecting itself. The potential role of male nursing students in promoting positive attitudes towards vaccination can be used as positive contributors to share positive vaccination experience with unvaccinated peers.

\subsection{Factors preventing participants from getting HPV vaccine}

\subsubsection{Low awareness rate}

Insufficient knowledge of HPV virus and nine valent HPV vaccine. Some studies have pointed out that, the main reason why college students are unwilling to receive HPV vaccine is that they don't understand the vaccine and think they don't have the risk of related diseases ${ }^{[9]}$. It was learned from the interview that some male nursing students were not clear about HPV virus. When asked who was most likely to be infected with HPV, 8 participants said that people with chaotic private life, sex workers, lack of attention to cleanliness and low immunity were easy to be infected. Participant C:'I think HPV infection is similar to HIV infection, and the susceptible people are those with unstable sexual partners. In addition, it is best not to smoke, which is easy to cause low immunity." Participant G: "Even boys should pay attention to local cleanliness, which is good for their girlfriends." When asked if he had any experience in HPV classroom education, (laughter) participant B said: "It seems that there was gynecology and obstetrics nursing in freshman year. At that time, the teacher only talked about HPV in class. After listening to the class, he felt that he should pay more attention to his personal hygiene and wash his underwear frequently, because it was not the focus of the exam, and he didn't pay much attention to this aspect later." Participant J: "I checked the human papillomavirus (HPV) on the Internet before, but I didn't see the introduction about the incidence of men, so I think it doesn't seem to be a problem for men (laughter)."

\subsubsection{Feminization of vaccine information}

Compared with the research on HPV infection and female reproductive health, the impact of HPV on male reproductive health has not been paid enough attention ${ }^{[10]}$. From the interview results, 10 male nursing students, even if they have heard of HPV and nine valent HPV vaccine, think that the vaccinated population is women and men do not need to be vaccinated. This is largely due to the widespread misunderstanding of the role of the vaccine. At the initial publicity stage, the vaccine was only sold to girls. Most people linked HPV infection with cervical cancer and thought that men had little to do with HPV. All 12 participants believed that HPV vaccination was mainly an intervention designed to prevent cervical cancer in girls or women. When asked if you think men need to be vaccinated with nine valent HPV vaccine, participant a expressed surprise: "Since HPV infection can lead to cervical cancer, men will not get this disease. Does it have anything to do with us?" Participant B: "Isn't the nine valent vaccine the cervical cancer vaccine? All female students in our class are discussing this matter. I don't think men need vaccination at all." Participant J: "I've heard of it (HPV vaccine) and it's still in the ads on those websites. However, most of the above are that HPV will affect women and cervical cancer, and we don't see anything that has an impact on men's health." 


\subsubsection{High vaccination cost}

Some participants made it clear that the high cost was the main factor leading them not to receive the nine valent HPV vaccine. Despite the great desire for vaccination, the obvious benefits and necessity of vaccination are still being weighed against major concerns about affordability. Participant I: "As a student, I have no source of income. Basically, my living expenses depend on school subsidies and the support of my family. I heard that the nine valent vaccine has a high coverage of the virus. I am also very eager to get vaccinated, but the vaccination cost is about 4000 yuan. Although I know that vaccination is beneficial to promoting my health, it is actually not cost-effective for men (helpless)." Participant E: "The nine-valent vaccine is expensive. If a doctor recommends it to me, I will doubt his purpose of giving me such an expensive vaccine, whether he can profit from it, etc. Besides, it is not a necessity for men. If doctors give us a vaccine like pneumonia and hepatitis B, we will certainly inoculate it for health reasons."

\subsubsection{Safety and effectiveness}

Some participants were concerned about the safety and efficacy of nine valent HPV vaccine. Common adverse reactions of nine valent HPV vaccine include: General adverse reactions such as pain, swelling, induration and pruritus after injection, and occasional dizziness and syncope. However, some distorted and untrue reports from the media have spread wrong information to the public, resulting in a low level of understanding of the safety and effectiveness of the vaccine, and most people are skeptical. The nine-valent vaccines have been on the market for a short time, and the long-term control effect is still unknown. Participant L: "I learned from consulting the literature that it takes more than ten years or even decades to develop from HPV infection to cervical cancer, while the nine valent vaccines have been on the market worldwide for only five years. At present, there is no clinical data at home and abroad that the vaccine is effective in preventing cervical cancer. I can only estimate theoretically whether vaccination can bring me long-term and effective protection." Participant C: "If the nine-valent vaccine really works as advertised in the press, why hasn't the country vigorously promoted the vaccine, included in medical insurance or given some preferential policies to encourage the vaccination of school-age people?"

\subsubsection{Imbalance between supply and demand}

Since the outbreak of novel coronavirus pneumonia, the nine valent HPV vaccine is seriously inadequate as an imported vaccine. The nine valent vaccine is difficult to make appointment and vaccination. Participant H: "When I looked through the circle of friends, I found that several female students and female friends were asking where to get the nine-valent vaccine. Some could not make an appointment after the first injection. If the vaccination could not meet the needs of women, even if I felt it necessary, I would consider delaying the injection." The vaccinated population is more passive, so as to reduce the vaccination rate.

\section{Discussion}

\subsection{Risk perception and cost independently and jointly affect the decision-making of male nursing students}

The feminization of HPV and nine valent HPV vaccine information affected the knowledge level of participants, leading many participants to believe that human papillomavirus vaccine has nothing to do with their health. According to the interview, male nursing students generally have low awareness of human papillomavirus and nine valent HPV vaccine, which is basically consistent with the survey in other regions in China ${ }^{[11]}$. Improving male nursing students' HPV related knowledge will greatly increase their willingness to vaccinate HPV vaccine. Male nursing students are an important group. They are future health professionals. They should have a certain understanding of human papillomavirus infection and vaccination. 
In addition, male nursing students also belong to the risk group in terms of human papillomavirus infection and related complications ${ }^{[12]}$. Only when male nursing students understand the harmfulness of HPV virus, such as persistent high-risk HPV virus infection may lead to cervical cancer and penile cancer, will they be willing to take the initiative to understand how to prevent these adverse outcomes. In addition, participants expressed concern about the high cost of imported nine-valent HPV vaccine and looked forward to developing domestic vaccine with high cost performance as soon as possible, so as to promote vaccination. Many participants said that before attending this interview, they had never seriously considered vaccination with nine-valent vaccine. They would consult their doctors about HPV vaccination at their next visit.

\subsection{The awareness rate of nine valent HPV vaccine increased with the increase of male nursing students' education}

This study shows that the level of HPV knowledge, HPV vaccine cognition and attitude of nursing master students is significantly higher than that of undergraduates and college students, which is consistent with the research of Ouyang lichen ${ }^{[13]}$ and Zeng Xiaomin ${ }^{[14]}$. When asked about HPV vaccine, what relevant knowledge do you want to know urgently? Most participants were interested in the number, time and place of vaccination. However, when asked where male nursing students will continue to acquire HPV related knowledge in the next step, college students and undergraduates said that they are more likely to accept medical lectures and special lectures, indicating that medical professionals play an important role in publicity and education activities. The postgraduate students prefer to consult the literature, which shows that the more information education they get, the higher their ability to integrate computer resources and absorb information.

\subsection{The promotion prospect of nine valent HPV vaccine in male nursing students has a long way to go}

The emergence of HPV vaccine has upgraded the prevention mode of cervical cancer. It is believed that cervical cancer can become the first cancer that can be completely eradicated through comprehensive preventive measures ${ }^{[15]}$. Improving the vaccination rate of nine valent vaccine can indirectly protect unvaccinated individuals through mass immunization, and encourage vaccination as a standard behavior. At present, the safety and effectiveness of the three vaccines listed in China have been verified by clinical trials. However, due to China's large population and limited awareness of HPV, and the nine valent HPV vaccine is not included in medical insurance as a bivalent vaccine, the vaccination rate is not ideal, and the promotion in China is facing great challenges. At present, the vaccination cost of nine valent vaccine is about 4000 yuan. As a developing country, if the vaccine is included in the immunization regulations, the economic burden will be heavy. Therefore, it is necessary to develop a cost-effective, safe and effective vaccine as soon as possible in combination with the actual situation of our country.

\section{Conclusion}

It is suggested that college education should use various forms and combined with the professional background of nursing to strengthen the knowledge of male nursing students about cervical cancer and preventive HPV vaccine, regularly popularize science on the bulletin board, and correct their incorrect information and behavior. The government should establish a systematic and scientific publicity mechanism in combination with the media to comprehensively and systematically publicize and educate the correct prevention and treatment knowledge of HPV infection. The focus of health education can focus on the effectiveness, safety and vaccination related knowledge of nine valent HPV vaccine, so as to improve men's cognition and vaccination willingness. At present, there is no effective solution to the imbalance between supply and demand of nine valent HPV vaccine. It is suggested that APP should be designed 
specifically, and WeChat official account should be combined to carry out HPV related knowledge propaganda and online appointment of HPV vaccine, so as to provide convenient inoculation for inoculant. This interview is limited to male nursing students. In the future, we can further study from the perspective of teachers and obstetricians to obtain more accurate information and improve the vaccination rate.

\section{Disclosure statement}

The author declares no conflict of interest.

\section{References}

[1] Iorga L, Dragos MR, Cristina DC, et al., 2020, Penile Carcinoma and HPV Infection (Review). Exp Ther Med. 20(1): 91-96.

[2] Wang R, Pan W, Jin L, et al., 2020, Human Papillomavirus Vaccine against Cervical Cancer: Opportunity and Challenge. Cancer Lett. 28(471):88-102.

[3] Canfell K, Kim JJ, Brisson M, et al., 2020, Mortality Impact of Achieving WHO Cervical Cancer Elimination Targets: A Comparative Modelling Analysis in 78 Low-Income and Lower-MiddleIncome Countries. Lancet. 22;395(10224):591-603.

[4] Skinner SR, Davies C, Cooper S, et al., 2015, HPV Edu Study Protocol:A Cluster Randomised Controlled Evaluation of Education, Decisional Support and Logistical Strategies in School-based Human Papillomavirus Vaccination (HPV) of Adolescents. BMC Public Health. 15(15): 896.

[5] Li P, Li Z, Qin Q, et al., 2017, Exploration and Practice of Training Mode for Male Nursing Students. General Nursing, 15(24): 3052-3054.

[6] Chen X, 2000, Qualitative Research Methods and Social Science Research. Beijing: Educational Science Press, 277-307.

[7] Liu M, 2008, Study on Nursing Quality. Beijing: People's Health Publishing House, 39-74.

[8] Smalley RC, Roller N, Pellom ST, et al., 2020, Therapeutic Vaccines for HPV-Associated Malignancies. Immunotargets Ther. 9:167-200.

[9] Li Y, 2017, Relationship between Human Papillomavirus Infection and Male Reproductive Health. Chinese Journal of Andrology, 23(04): 376-380.

[10] Zulibiyan I, 2018, Investigation on the Awareness of some Female College Students about Cervical Cancer and HPV in Urumqi, Xinjiang. Urumqi: Xinjiang Medical University.

[11] Pang Z, Chen X, Xiang Y, 2016, Investigation and Analysis on Influencing Factors of Female College Students' Cognition and Vaccination Intention of Human Papillomavirus Vaccine in Guangzhou University City. Research and Practice of Health Medicine, 13 (02): 22-24.

[12] Wu Q, Xu Y, Zhang J, et al., 2018, Human Papillomavirus Awareness and Vaccination Intention of Female Medical Students in Guangzhou. China School Health, 39(11): 1699-1701.

[13] Ouyang L, Wang S, et al., 2020, Investigation on Female Medical Students' Cognition and Vaccination Willingness of HPV and its Vaccine in Jianghan University. Journal of Jianghan University (Natural Science Edition), 48(05): 47-54.

[14] Zeng X, Ren Z, Wei X, et al., 2015, Investigation on the Cognition of HPV and Vaccine among Undergraduates of Sun Yat Een University. Modern Preventive Medicine, 42 (10): 1822-1825.

[15] Zhang J, Han Y, Wang R, et al., 2020, Investigation on Knowledge, Belief and Practice of Human Papillomavirus and HPV Vaccine in Different Populations. China Health Education, 36 (02): 147152. 